\title{
Characterization, Classification and Nutritional Status of Groundnut Growing Soils in Southern Agro-Climatic Zone: A Case Study on Western Mandals of Srikalahasti Division in Chittoor District, Andhra Pradesh, India
}

V. Nagarjuna* and M.V.S. Naidu

\author{
Department of Soil Science and Agricultural Chemistry, S. V.Agricultural College, Acharya \\ N.G.Ranga Agricultural University, Tirupati - 517502, Andhra Pradesh, India
}

*Corresponding author

\begin{tabular}{|l|}
\hline K e y w o r d s \\
Characterization, \\
Classification, \\
Groundnut-growing soils, \\
Nutrient status, \\
Srikalahasti division
\end{tabular}

\section{Introduction}

Groundnut (Arachis hypogaea L.) is the most popular oilseed crop in India. This is known as Peanut, Earthnut, Monkey nut, Goober panda and Manilanut and contains about 45 percent oil and 26 percent protein. Groundnut kernels as a whole are highly digestible. One gram of kernel supplies 5.8 calories of food. This is compared with four calories per gram of sugar, 3.5 calories for whole wheat and 2.6 calories of bread. The biological value of groundnut protein is among the highest of the vegetable proteins and equal to that of casein. 
India has prime position in average area (5.31 million ha) and production (7.17 million tonnes) of groundnut. In Andhra Pradesh, it is cultivated in an area of about 13.86 lakh ha with an annual production of 12.34 lakh tonnes. India stands first in area and second in production and fifth in productivity. The reason for low productivity $\left(1305 \mathrm{~kg} \mathrm{ha}^{-1}\right)$ of groundnut in India as compared to other countries like USA (3000 kg ha ${ }^{-1}$ ) and China $\left(2600 \mathrm{~kg} \mathrm{ha}^{-1}\right)$ are mainly due to rain dependency (85\%), monoculture $(60 \%)$ and cultivation on marginal soils of low fertility.

Comprehensive knowledge on soil resources in terms of types of soil, their spatial extent, physical and chemical properties and limitations or capabilities for proper management to sustain soil productivity and crop yields is highly essential. Though groundnut is a commercial crop, till now the groundnut-growing soils were neither characterized and classified nor studied for nutrient status in particular in Srikalahasti division in Chittoor district and in general in Andhra Pradesh. Hence, the present investigation was carried out.

\section{Materials and Methods}

\section{Study area}

The study area lies in between $13^{0} 25^{\prime}$ and $14^{\circ} 05^{\prime} \mathrm{N}$ latitude and $79^{\circ} .12^{\prime}$ and $80^{\circ} .08^{\prime}$ longitude. It represents semi-arid monsoonic climate with distinct summer, winter and rainy seasons. The annual precipitation was 888.44 $\mathrm{mm}$ of which 94.21 per cent was received during May to December. The mean annual soil temperature was $27.66^{\circ} \mathrm{C}$ with mean summer and winter temperatures of 31.79 and $27.06^{\circ} \mathrm{C}$, respectively. The area qualifies for isohyperthermic temperature regime. The soil moisture control section remains dry for more than 90 cumulative days or 45 consecutive days in four months following summer solistice and this qualifies for ustic soil moisture regime. The natural vegetation of the study area was Parthenium hysterophorus, Calotropis gigantia, Tridax procumbens, Pongamia pinnata, Azardirachta indica, Lantana camera, Cyperus rotundus and Cynodon dactylon. The soils were developed from granite-gneiss and alluvium parent materials.

\section{Methods}

A reconnaissance soil survey was conducted in groundnut growing soils of Srikalahasti division in Chittoor district as per the procedure outlined by AIS \& LUS (1970) on 1:50,000 scale. Eight typical pedons i.e., one pedon in plains and seven in uplands were studied for their morphological characteristics following the procedure outlined by Soil Survey Staff (1951). Later, horizon-wise soil samples were collected and analyzed for their physical and physico-chemical properties and available nutrient status using standard procedures and classified according to Soil Taxonomy (Soil Survey Staff, 2014). The soil samples were classified into low, medium and high categories as per limits suggested by Muhr et al., (1965) for available N, P and K and organic carbon. Available sulphur was rated based on the limits proposed by Tandon (1991). In respect of available micronutrients, the ratings given by Lindsay and Norvell (1978) were followed.

\section{Results and Discussion}

\section{Morphological properties}

The site and morphometric characteristics of the pedons have been given in table 1 and 2 , respectively. The pedons had their munsell colour notation in the hue of $10 \mathrm{YR}$ barring pedon P2, P4 and P8 (7.5YR), value 2 to 6 and chroma 1 to 6 (Table 2). The dark colour of these soils may be attributed to domination of 
highly dispersed form of humus and smectite minerals (Zonn 1986). The dominant soil structure was fine to medium, weak to moderate and crumb in surface and subangular blocky in sub-surface horizons.

\section{Physical properties}

The clay content of the pedons ranged from 9.44 to 37.12 per cent (Table 3). There was lithological discontinuity in $\mathrm{P}_{2}, \mathrm{P}_{3}$ and $\mathrm{P}_{5}$ as evidenced from marked variation in sand/silt ratio (Ray and Reddy 1997). In general silt content ranged from 2.22 to 28.25 per cent. Bulk density of the groundnut-growing soils ranged from 1.26 to $1.60 \mathrm{Mg} \mathrm{m}^{-3}$. Since groundnut-growing soils are tilled up to 15-20 $\mathrm{cm}$ deep during planting and disintegration of aggregates and soil structure through tillage can result in increased bulk density with depth. Similar results were reported by Six et al., (1998). Higher bulk density values in the sub-surface could be ascribed to decreased organic matter and secondary accumulation of illuviated clay in pore space. Bulk density decreased in horizons just below the Ap layer and then increased with depth in pedons 4,7 and 8 which could be attributed to decreased organic matter content and secondary accumulation of illuviated clays, but further orientation of clay in pore space increased the bulk density value in $\mathrm{C}$ horizon. The water holding capacity was positively and significantly correlated with clay $(\mathrm{r}=$ $+0.750 *$.

\section{Physico-chemical properties}

The $\mathrm{pH}$ of the soils ranged from 6.61 (near neutral) to 8.14 (slightly alkaline) (Table 4). Electrical conductivity of soils varied from 0.01 to $0.04 \mathrm{dSm}^{-1}$ indicating the non-saline nature of soils. Organic carbon content of the soils ranged from 0.09 (low) to 0.58 (medium) per cent and decreased with depth which could be attributed to the addition of plant residues and farm yard manure to surface horizons than in the lower horizons. The $\mathrm{CaCO}_{3}$ content of these soils ranged from 2.40 to 3.23 per cent. In pedons 3 and 4 the $\mathrm{CaCO}_{3}$ increased with depth which might be due to down ward movement of calcium and its subsequent precipitation as carbonate and / or decomposition of calcium carbonate.

In general $\mathrm{Ca}^{+2}$ is the dominant cation on exchange complex followed by $\mathrm{Mg}^{+2}, \mathrm{Na}^{+}$and $\mathrm{K}^{+}$. Cation exchange capacity of the soils ranged between 12.15 to $46.26 \mathrm{cmol}\left(\mathrm{p}^{+}\right) \mathrm{kg}^{-1}$ in different horizons and was positively and significantly correlated with clay $(\mathrm{r}=$ $\left.+0.721^{* *}\right)$ and was negatively and significantly correlated with sand $(\mathrm{r}=$ $\left.0.462^{* *}\right)$. The base saturation ranged from 57.02 to 90.14 per cent (Table 4). The higher base saturation in some pedons might be due to higher amount of $\mathrm{Ca}^{+2}$ occupying exchange sites on the colloidal complex and also may be due to recycling of basic cations through vegetation.

\section{Nutrient status and soil fertility}

\section{Macronutrients}

The available $\mathrm{N}$ in soils was low ranging from $72.80\left(\mathrm{P}_{1}\right)$ to $185.10 \mathrm{mg} \mathrm{kg}^{-1}\left(\mathrm{P}_{2}\right)$ (Table 5) and found to be maximum in surface horizons and decreased with depth which is due to decreasing trend of organic carbon with depth. Further, the available nitrogen was significantly and positively correlated $(\mathrm{r}=$ $+0.640 * *)$ with organic carbon. Low available $\mathrm{N}$ status in these soils might be due to the fact that, semi-arid condition of the area might have favoured rapid oxidation and lesser accumulation of organic matter, releasing more $\mathrm{NO}_{3}-\mathrm{N}$ which could have been lost by leaching (Finck and Venkateswarlu, 1982). The available $\mathrm{P}$ was low to high and varied from 5.00 to $15.80 \mathrm{mg} \mathrm{kg}^{-1}$ and decreased with depth. 
Table.1 Salient site features of the profiles studied

\begin{tabular}{|c|c|c|c|c|c|c|c|c|}
\hline Features & $\begin{array}{c}\text { Pedon } \\
\text { (Suryanaraynapuram *) }\end{array}$ & $\begin{array}{c}\text { Pedon 2 } \\
\text { (Kallivettu *) }\end{array}$ & $\begin{array}{c}\text { Pedon 3 } \\
\text { (Gajulapellore *) }\end{array}$ & $\begin{array}{c}\text { Pedon } 4 \\
\text { (Kanamanambedu *) }\end{array}$ & \begin{tabular}{c|} 
Pedon 5 \\
$($ Kalathuru *)
\end{tabular} & $\begin{array}{c}\text { Pedon 6 } \\
\text { (Thimmasamudram *) }\end{array}$ & $\begin{array}{c}\text { Pedon } 7 \\
\text { (Chittathur*) }\end{array}$ & $\begin{array}{c}\text { Pedon 8 } \\
\text { (Brahmanapalle*) }\end{array}$ \\
\hline Physiography & Plain & Upland & Upland & Upland & Upland & Upland & Upland & Upland \\
\hline Slope (\%) & $0-1$ & $1-3$ & $1-3$ & $1-3$ & $0-1$ & $1-3$ & $3-5$ & $3-5$ \\
\hline Elevation (msl) & $97 \mathrm{~m}$ & $29 m$ & $21 \mathrm{~m}$ & $70 \mathrm{~m}$ & $113 \mathrm{~m}$ & $71 \mathrm{~m}$ & $49 \mathrm{~m}$ & $66 \mathrm{~m}$ \\
\hline Drainage & $\begin{array}{l}\text { Moderately well } \\
\text { drained }\end{array}$ & $\begin{array}{l}\text { Moderately } \\
\text { well drained }\end{array}$ & Well drained & Well drained & $\begin{array}{c}\text { Moderately } \\
\text { well drained }\end{array}$ & Well drained & Well drained & Well drained \\
\hline $\begin{array}{c}\text { Parent } \\
\text { material }\end{array}$ & Granite-gneiss & Granite-gneiss & Granite-gneiss & Granite-gneiss & $\begin{array}{l}\text { Granite- } \\
\text { gneiss }\end{array}$ & Granite-gneiss & Granite-gneiss & Granite-gneiss \\
\hline Erosion & Very slight & Slight & Slight & Slight & Very slight & Slight & Moderate & Moderate \\
\hline Land use & Groundnut crop & $\begin{array}{l}\text { Groundnut } \\
\text { crop }\end{array}$ & Groundnut crop & Groundnut crop & $\begin{array}{l}\text { Groundnut } \\
\text { crop }\end{array}$ & Groundnut crop & $\begin{array}{l}\text { Groundnut } \\
\text { crop }\end{array}$ & Groundnut crop \\
\hline
\end{tabular}

*Name of the village 
Table.2 Summary of the morphological characters of the pedons

\begin{tabular}{|c|c|c|c|c|c|c|c|c|c|c|c|c|c|c|c|c|c|c|c|c|c|}
\hline \multirow{2}{*}{$\begin{array}{l}\text { Pedon No. } \\
\text { and Horizon }\end{array}$} & \multirow[t]{2}{*}{ Depth (m) } & \multicolumn{2}{|c|}{ Colour } & \multirow[t]{2}{*}{ Texture } & \multicolumn{3}{|c|}{ Structure } & \multicolumn{3}{|c|}{ Consistence } & \multirow[t]{2}{*}{ Efferve-scence } & \multicolumn{2}{|c|}{ Boundary } & \multicolumn{3}{|c|}{ Cutans } & \multicolumn{2}{|c|}{ Pores } & \multicolumn{2}{|c|}{ Roots } & \multirow[t]{2}{*}{ Other features } \\
\hline & & Moist & Dry & & $\mathbf{S}$ & G & $\mathbf{T}$ & Dry & $\begin{array}{c}\text { Mois } \\
\mathbf{t}\end{array}$ & $\begin{array}{c}\text { We } \\
\mathbf{t}\end{array}$ & & D & $\mathbf{T}$ & $\begin{array}{l}\mathbf{T} \\
\mathbf{Y}\end{array}$ & $\begin{array}{l}\mathbf{T} \\
\mathbf{H}\end{array}$ & Q & $\mathbf{S}$ & $\mathbf{Q}$ & $\mathbf{S}$ & $\mathbf{Q}$ & \\
\hline \multicolumn{22}{|l|}{ Pedon 1} \\
\hline Ap & $0.00-0.20$ & $10 \mathrm{YR} 4 / 2$ & 10 YR 5/2 & sl & $\mathrm{f}$ & 1 & $\mathrm{cr}$ & $\mathrm{sh}$ & $\mathrm{fr}$ & ssps & - & $\mathrm{c}$ & $\mathrm{s}$ & - & - & - & - & - & $\mathrm{f}$ & $\mathrm{f}$ & \\
\hline $\mathbf{A 1}$ & $0.20-0.40$ & $10 \mathrm{YR} 4 / 4$ & 10 YR 5/6 & sl & $\mathrm{f}$ & 1 & sbk & $\mathrm{sh}$ & $\mathrm{fr}$ & ssps & - & $\mathrm{c}$ & $\mathrm{s}$ & - & - & - & - & - & $\mathrm{f}$ & $\mathrm{f}$ & Fe \& Mn Nodules \\
\hline Bw1 & $0.40-0.69$ & 10 YR 5/6 & 10 YR 6/6 & $\mathrm{scl}$ & $\mathrm{m}$ & 2 & sbk & $\mathrm{h}$ & fi & $\mathrm{sp}$ & - & $\mathrm{c}$ & $\mathrm{s}$ & - & - & - & - & - & - & - & Fe \& Mn Nodules \\
\hline Bw2 & $0.69-0.90$ & $10 \mathrm{YR} 6 / 3$ & $10 \mathrm{YR} 7 / 3$ & scl & $\mathrm{m}$ & 2 & sbk & $\mathrm{h}$ & fi & $\mathrm{sp}$ & - & d & w & - & - & - & - & - & - & - & Fe \& Mn Nodules \\
\hline Bw3 & $0.90-1.10$ & $10 \mathrm{YR} 7 / 4$ & $10 \mathrm{YR} 8 / 2$ & $\mathrm{scl}$ & $\mathrm{m}$ & 2 & sbk & $\mathrm{h}$ & fi & $\mathrm{sp}$ & - & $\mathrm{d}$ & w & - & - & - & - & - & - & - & Fe \& Mn Nodules \\
\hline \multicolumn{22}{|l|}{ Pedon 2} \\
\hline Ap & $0.00-0.18$ & 7.5 YR 4/1 & $\begin{array}{c}7.5 \mathrm{YR} \\
6 / 1\end{array}$ & sl & $\mathrm{f}$ & 1 & $\mathrm{cr}$ & $\operatorname{sh}$ & fr & ssps & - & $\mathrm{c}$ & $\mathrm{s}$ & - & - & - & - & - & $\mathrm{f}$ & $\mathrm{f}$ & \\
\hline$\overline{\text { Bw1 }}$ & $0.18-0.55$ & $10 \mathrm{YR} 4 / 2$ & 10 YR 6/2 & $\mathrm{scl}$ & $\mathrm{m}$ & 2 & sbk & $\mathrm{h}$ & fi & $\mathrm{sp}$ & - & $\mathrm{c}$ & $\mathrm{s}$ & - & - & - & - & - & $\mathrm{f}$ & $\mathrm{f}$ & \\
\hline Bw2 & $0.55-0.84$ & $10 \mathrm{YR} 4 / 3$ & 10 YR 6/3 & sl & $\mathrm{f}$ & 1 & sbk & $\mathrm{h}$ & fi & ssps & - & $\mathrm{d}$ & w & - & - & - & - & - & - & - & \\
\hline Bw3 & $0.84-1.02$ & $10 \mathrm{YR} 4 / 2$ & 10 YR 6/3 & $\mathrm{sc}$ & $\mathrm{c}$ & 3 & abk & $\mathrm{h}$ & fi & $\begin{array}{l}\text { vsv } \\
\mathrm{p}\end{array}$ & - & d & w & - & - & - & - & - & - & - & \\
\hline $\mathrm{Cr}$ & \multicolumn{21}{|c|}{ Weathered gneiss mixed with lime } \\
\hline \multicolumn{22}{|l|}{ Pedon 3} \\
\hline $\mathbf{A p}$ & $0.00-0.22$ & $10 \mathrm{YR} 3 / 6$ & 10 YR 5/4 & $\mathrm{scl}$ & $\mathrm{f}$ & 1 & $\mathrm{cr}$ & $\mathrm{sh}$ & $\mathrm{fr}$ & ssps & - & $\mathrm{c}$ & $\mathrm{s}$ & - & - & - & - & - & $\mathrm{f}$ & $\mathrm{f}$ & \\
\hline A1 & $0.22-0.33$ & 10 YR 3/4 & 10 YR 5/6 & sl & $\mathrm{f}$ & 1 & sbk & sh & $\mathrm{fr}$ & ssps & - & d & w & - & - & - & - & - & f & $\mathrm{f}$ & \\
\hline $\mathbf{R}$ & \multicolumn{21}{|l|}{ Hard Rock } \\
\hline \multicolumn{22}{|l|}{ Pedon 4} \\
\hline Ap & $0.00-0.15$ & $5 \mathrm{YR} 4 / 3$ & 5 YR 5/6 & $\mathrm{scl}$ & f & 2 & $\mathrm{cr}$ & $\mathrm{sh}$ & fi & ssps & - & $\mathrm{c}$ & S & - & - & - & - & - & $\mathrm{f}$ & f & \\
\hline Bt1 & $0.15-0.34$ & 7.5 YR 3/3 & $\begin{array}{c}7.5 \mathrm{YR} \\
4 / 6\end{array}$ & $\mathrm{scl}$ & $\mathrm{m}$ & 2 & sbk & $\mathrm{h}$ & fi & $\mathrm{sp}$ & - & $\mathrm{c}$ & $\mathrm{s}$ & - & - & - & - & - & $\mathrm{f}$ & $\mathrm{f}$ & \\
\hline Bt2 & $0.34-0.80$ & 7.5 YR 3/4 & $\begin{array}{c}7.5 \mathrm{YR} \\
4 / 4\end{array}$ & sc & $\mathrm{m}$ & 3 & abk & $\mathrm{h}$ & fi & $\mathrm{sp}$ & - & $\mathrm{d}$ & w & - & - & - & - & - & - & - & \\
\hline $\mathrm{Cr}$ & \multicolumn{21}{|c|}{ Weathered gneiss } \\
\hline \multicolumn{22}{|l|}{ Pedon 5} \\
\hline Ap & $0.00-0.23$ & $10 \mathrm{YR} 4 / 2$ & 10 YR 5/2 & sl & $\mathrm{f}$ & 1 & $\mathrm{cr}$ & $\mathrm{sh}$ & $\mathrm{fr}$ & ssps & - & $\mathrm{c}$ & $\mathrm{s}$ & - & - & - & - & - & $\mathrm{f}$ & $\mathrm{f}$ & \\
\hline A1 & $0.23-0.44$ & 10 YR $3 / 3$ & 10 YR 5/3 & sl & $\mathrm{f}$ & 1 & sbk & $\mathrm{h}$ & $\mathrm{fr}$ & ssps & - & $\mathrm{c}$ & $\mathrm{s}$ & - & - & - & - & - & $\mathrm{f}$ & $\mathrm{f}$ & \\
\hline $\mathbf{A 2}$ & $0.44-0.69$ & 10 YR 4/4 & 10 YR 5/4 & $\mathrm{scl}$ & $\mathrm{m}$ & 2 & sbk & $\mathrm{h}$ & fi & $\mathrm{sp}$ & - & $\mathrm{d}$ & w & - & - & - & - & - & - & - & Fe \& Mn mottles \\
\hline A3 & $0.69-0.92$ & $10 \mathrm{YR} 4 / 4$ & 10 YR 5/6 & sl & $\mathrm{m}$ & 2 & sbk & $\mathrm{h}$ & $\mathrm{fr}$ & sssp & - & $\mathrm{d}$ & w & - & - & - & - & - & - & - & Fe \& Mn mottles \\
\hline A4 & $0.92-1.30+$ & 10 YR 5/4 & 10 YR 6/2 & $\mathrm{scl}$ & $\mathrm{m}$ & 2 & sbk & $\mathrm{h}$ & fi & $\mathrm{sp}$ & - & $\mathrm{d}$ & w & - & - & - & - & - & - & - & \\
\hline
\end{tabular}




\begin{tabular}{|c|c|c|c|c|c|c|c|c|c|c|c|c|c|c|c|c|c|c|c|c|c|}
\hline \multirow{3}{*}{$\begin{array}{c}\text { Pedon No. } \\
\& \\
\text { Horizon }\end{array}$} & & & & & & & & & & & & & & & & & & & & & t... \\
\hline & \multirow[t]{2}{*}{ Depth (m) } & \multicolumn{2}{|c|}{ Colour } & \multirow[t]{2}{*}{ Texture } & \multicolumn{3}{|c|}{ Structure } & \multicolumn{3}{|c|}{ Consistence } & \multirow{2}{*}{$\begin{array}{l}\text { Efferve- } \\
\text { scence }\end{array}$} & \multicolumn{2}{|c|}{ Boundary } & \multicolumn{3}{|c|}{ Cutans } & \multicolumn{2}{|c|}{ Pores } & \multicolumn{2}{|c|}{ Roots } & \multirow{2}{*}{$\begin{array}{l}\text { Other } \\
\text { features }\end{array}$} \\
\hline & & Moist & Dry & & $\mathbf{S}$ & $\mathbf{G}$ & $\mathbf{T}$ & $\begin{array}{c}\text { Dr } \\
\mathbf{y}\end{array}$ & Moist & Wet & & D & $\mathbf{T}$ & $\begin{array}{l}\mathbf{T} \\
\mathbf{Y}\end{array}$ & $\begin{array}{l}\mathbf{T} \\
\mathbf{H}\end{array}$ & $\mathbf{Q}$ & $\mathbf{S}$ & $\mathbf{Q}$ & $\mathbf{S}$ & $\mathbf{Q}$ & \\
\hline \multicolumn{22}{|l|}{ Pedon 6} \\
\hline$\overline{A p}$ & $0.00-0.20$ & $10 \mathrm{YR} 4 / 2$ & 10 YR 6/3 & sl & $\mathrm{f}$ & 1 & $\mathrm{cr}$ & sh & fr & ssps & - & c & $\mathrm{s}$ & - & - & - & - & - & $\mathrm{f}$ & $\mathrm{f}$ & \\
\hline$\overline{\mathrm{A1}}$ & $0.20-0.54$ & 10YR 4/3 & 10 YR 6/2 & sl & $\mathrm{m}$ & 2 & sbk & sh & fr & ssps & - & c & $\mathrm{s}$ & - & - & - & - & - & $\mathrm{f}$ & $\mathrm{f}$ & \\
\hline Bw1 & $0.54-0.84$ & 10 YR 4/2 & 10 YR 6/4 & scl & $\mathrm{m}$ & 2 & sbk & $\mathrm{h}$ & fi & $\mathrm{sp}$ & - & c & $\mathrm{s}$ & - & - & - & - & - & - & - & \\
\hline Bw2 & $0.84-1.07$ & $10 \mathrm{YR} 4 / 3$ & 10 YR $6 / 3$ & sl & $\mathrm{m}$ & 2 & sbk & sh & fi & ssps & - & d & w & - & - & - & - & - & - & - & \\
\hline Bw3 & $1.07-1.50+$ & 10YR 4/2 & 10 YR 6/6 & $\mathrm{scl}$ & $\mathrm{m}$ & 2 & sbk & $\mathrm{h}$ & fi & $\mathrm{sp}$ & - & d & w & - & - & - & - & - & - & - & \\
\hline \multicolumn{22}{|l|}{ Pedon 7} \\
\hline Ap & $0.00-0.22$ & 10 YR 4/4 & 10 YR 5/8 & sl & $\mathrm{f}$ & 1 & $\mathrm{cr}$ & sh & fr & ssps & - & c & s & - & - & - & - & - & $\mathrm{f}$ & $\mathrm{f}$ & \\
\hline $\mathbf{A 1}$ & $0.22-0.46$ & 10 YR 3/6 & 10 YR 5/6 & sl & $\mathrm{m}$ & 2 & sbk & $\mathrm{h}$ & fi & ssps & - & $\mathrm{d}$ & w & - & - & - & - & - & f & $\mathrm{f}$ & \\
\hline$\overline{A 2}$ & $0.46-0.81$ & $10 \mathrm{YR} 3 / 3$ & 10 YR 5/8 & sl & $\mathrm{m}$ & 2 & sbk & $\mathrm{h}$ & fr & ssps & - & d & w & - & - & - & - & - & - & - & \\
\hline $\mathrm{Cr}$ & \multicolumn{20}{|c|}{ Weathered gneiss mixed with soil } & \\
\hline Pedon 8 & & & & & & & & & & & & & & & & & & & & & \\
\hline Ap & $0.00-0.16$ & $\begin{array}{c}7.5 \mathrm{YR} \\
4 / 4\end{array}$ & $\begin{array}{c}7.5 \mathrm{YR} \\
6 / 8\end{array}$ & $\mathrm{scl}$ & $\mathrm{f}$ & 1 & $\mathrm{cr}$ & $\operatorname{sh}$ & fr & ssps & - & $\mathrm{c}$ & $\mathrm{s}$ & - & - & - & - & - & f & $\mathrm{f}$ & \\
\hline$\overline{\mathbf{A 1}}$ & $0.16-0.34$ & 10 YR 4/4 & 10 YR 6/8 & sl & $\mathrm{f}$ & 1 & sbk & sh & fr & ssps & - & d & w & - & - & - & - & - & $\mathrm{f}$ & $\mathrm{f}$ & \\
\hline$\overline{A 2}$ & $0.34-0.58$ & $10 \mathrm{YR} 4 / 4$ & 10 YR 6/6 & scl & $\mathrm{m}$ & 2 & sbk & sh & fr & ssps & - & d & w & - & - & - & - & - & - & - & \\
\hline $\mathrm{Cr}$ & \multicolumn{21}{|c|}{ Weathered gneiss mixed with soil } \\
\hline
\end{tabular}

Texture: $\mathrm{c}$ - clay, cl - clay loam, 1 - loam, s - sand, sl - sandy loam, scl - sandy clay loam, sc - sandy clay, ls - loamy sand

Structure: Size (S) - vf - very fine, f - fine, $\mathrm{m}$ - medium, c - coarse; Grade $(\mathrm{G})$ - O - structureless, 1 - weak, 2 - moderate, 3 - strong; Type (T) : cr - crumb, sg - single grain, abk - angular blocky, sbk - sub-angular blocky.Consistence:

Dry: s - soft, 1 - loose, sh - slightly hard, h - hard, vh - very hard

Moist: 1 - loose, fr - friable, fi - firm, vfi - very firm

Wet: so - non-sticky, ss - slightly sticky, s - sticky, vs - very sticky; po - non-plastic, ps - slightly plastic, p - plastic, vp - very plastic

Cutans: Ty - type: $\mathrm{t}$ - argillan, Th - Thickness: $\mathrm{tn}-$ thin, th - thick; Quantity $(\mathrm{Q}): \mathrm{p}-$ patchy, $\mathrm{c}-$ continuous

Pores: Size (S): $\mathrm{f}$ - fine, $\mathrm{m}$ - medium, c- coarse; Q: Quantity, $\mathrm{f}$ - few, c - common, $\mathrm{m}$ - many

Roots: Size (S): $\mathrm{f}$ - fine, $\mathrm{m}$ - medium, c- coarse; $\mathrm{Q}$ : Quantity, $\mathrm{f}$ - few, c - common, $\mathrm{m}$ - many

Effervescence: es - strong effervescence, ev - violent effervescence

Boundary: D: distinctness, $\mathrm{c}$ - clear, $\mathrm{g}$ - gradual, $\mathrm{d}$ - diffuse; $\mathrm{T}$ - Topography: $\mathrm{s}$ - smooth, $\mathrm{w}$ - wavy 
Table.3 Physical characteristics of the groundnut-growing soils

\begin{tabular}{|c|c|c|c|c|c|c|c|c|}
\hline $\begin{array}{c}\text { Pedon } \\
\text { No. \& } \\
\text { Horizon }\end{array}$ & $\begin{array}{c}\text { Depth } \\
\text { (m) }\end{array}$ & $\begin{array}{c}\text { Sand } \\
(\%) \\
(0.05- \\
2.0 \mathrm{~mm})\end{array}$ & $\begin{array}{c}\text { Silt (\%) } \\
(0.002- \\
0.05 \\
\mathrm{~mm})\end{array}$ & $\begin{array}{c}\text { Clay } \\
(\%) \\
(<0.002 \\
\text { mm })\end{array}$ & $\begin{array}{l}\text { Textural } \\
\text { class }\end{array}$ & $\begin{array}{l}\text { Sand } \\
\text { / Silt }\end{array}$ & $\begin{array}{c}\text { Bulk } \\
\text { density } \\
\left(\mathrm{Mg} \mathrm{m}^{-3}\right)\end{array}$ & $\begin{array}{l}\text { Water holding } \\
\text { capacity (\%) }\end{array}$ \\
\hline
\end{tabular}

Pedon 1 Suryanarayanapuram (Fine-loamy, smectitic, isohyperthermic, Typic Haplustept)

\begin{tabular}{|c|c|c|c|c|c|c|c|c|}
\hline Ap & $0.00-0.20$ & 74.79 & 15.41 & 9.80 & sl & 4.85 & 1.28 & 21.08 \\
\hline A1 & $0.20-0.40$ & 78.42 & 6.47 & 15.11 & sl & 12.12 & 1.29 & 37.46 \\
\hline Bw1 & $0.40-0.69$ & 69.15 & 5.71 & 25.14 & scl & 12.11 & 1.32 & 39.33 \\
\hline Bw2 & $0.69-0.90$ & 68.51 & 5.62 & 25.87 & scl & 12.19 & 1.26 & 39.73 \\
\hline Bw3 & $0.90-1.10$ & 62.21 & 10.00 & 27.79 & scl & 6.22 & 1.30 & 40.78 \\
\hline
\end{tabular}

Kallivettu (Fine-loamy, smectitic, isohyperthermic, Typic Haplustept)

\begin{tabular}{|c|c|c|c|c|c|c|c|c|}
\hline Ap & $0.00-0.18$ & 76.38 & 8.22 & 15.40 & sl & 9.29 & 1.51 & 29.38 \\
\hline Bw1 & $0.18-0.55$ & 73.20 & 3.66 & 23.14 & scl & 20.00 & 1.56 & 35.10 \\
\hline Bw2 & $0.55-0.84$ & 58.04 & 24.40 & 17.56 & sl & 2.38 & 1.49 & 33.90 \\
\hline Bw3 & $0.84-1.02$ & 48.21 & 14.67 & 37.12 & sc & 3.29 & 1.44 & 44.44 \\
\hline Cr & 1.02 & Weathered gneiss mixed with lime & & & \\
\hline
\end{tabular}

Gajulapellore (Coarse-loamy, siliceous, isohyperthermic, Lithic Ustorthent)

Ap

A1

$\mathbf{R}$

Pedon 4

Ap

Bt1

Bt2

$\mathrm{Cr}$

Pedon 5

Ap

A1

A2

A3

A4

Pedon 6

Ap

A1

Bw1

Bw2

Bw3

Pedon 7

Ap

A1

A2

$\mathrm{Cr}$

Pedon 8

Ap

A1

A2

$\mathrm{Cr}$

0.00-0.22

$0.22-0.33$

0.33

60.86

82.73

19.07

3.22

Hard Rock

Kanamanambedu (Fine-loamy, kaolinitic, isohyperthermic, Typic Haplustalf)

\begin{tabular}{|l|c|c|c|c|c|c|c|}
\hline $0.00-0.15$ & 73.43 & 5.08 & 21.49 & scl & 14.45 & 1.51 & 34.38 \\
\hline $0.15-0.34$ & 68.37 & 4.69 & 26.94 & scl & 14.58 & 1.33 & 35.27 \\
\hline $0.34-0.80$ & 56.63 & 8.24 & 35.13 & sc & 6.87 & 1.55 & 45.25 \\
\hline
\end{tabular}

Kalathuru (Fine-loamy, siliceous, isohyperthermic, Typic Ustrorthent)

\begin{tabular}{|c|c|c|c|c|c|c|c|}
\hline $0.00-0.23$ & 82.78 & 2.22 & 15.00 & sl & 37.29 & 1.36 & 29.12 \\
\hline $0.23-0.44$ & 76.90 & 4.20 & 18.90 & sl & 18.31 & 1.59 & 38.02 \\
\hline $0.44-0.69$ & 71.39 & 4.24 & 24.37 & scl & 16.84 & 1.42 & 52.19 \\
\hline $0.69-0.92$ & 53.49 & 28.25 & 18.26 & sl & 1.89 & 1.44 & 32.82 \\
\hline $0.92-1.30+$ & 67.03 & 10.99 & 21.98 & scl & 6.10 & 1.34 & 46.07 \\
\hline
\end{tabular}

Thimmasamudram (Coarse-loamy, smectitic, isohyperthermic, Typic Haplustept)

\begin{tabular}{|c|c|c|c|c|c|c|c|}
\hline $0.00-0.20$ & 77.57 & 12.99 & 9.44 & sl & 5.97 & 1.52 & 26.63 \\
\hline $0.20-0.54$ & 73.58 & 13.96 & 12.46 & sl & 5.27 & 1.54 & 28.85 \\
\hline $0.54-0.84$ & 65.54 & 13.14 & 21.32 & scl & 4.99 & 1.45 & 35.38 \\
\hline $0.84-1.07$ & 72.19 & 10.20 & 17.61 & sl & 7.08 & 1.59 & 33.63 \\
\hline $1.07-1.50+$ & 56.13 & 12.48 & 31.39 & scl & 4.50 & 1.44 & 38.05 \\
\hline
\end{tabular}

Chittathur (Coarse-loamy, siliceous, isohyperthermic, Typic Ustrorthent)

\begin{tabular}{|c|c|c|c|c|c|c|c|}
\hline $0.00-0.22$ & 72.78 & 7.78 & 19.44 & sl & 9.35 & 1.60 & 31.81 \\
\hline $0.22-0.46$ & 74.07 & 7.38 & 18.55 & sl & 10.04 & 1.47 & 30.80 \\
\hline $0.46-0.81$ & 81.14 & 2.02 & 16.84 & sl & 40.17 & 1.60 & 29.67 \\
\hline 0.81 & \multicolumn{7}{|c|}{ Weathered gneiss mixed with soil }
\end{tabular}

Brahmanapalle (Coarse-loamy, siliceous, isohyperthermic, Typic Ustrorthent)

\begin{tabular}{|c|c|c|c|c|c|c|c|}
\hline $0.00-0.16$ & 65.54 & 13.14 & 21.32 & scl & 4.99 & 1.40 & 30.18 \\
\hline $0.16-0.34$ & 63.55 & 21.87 & 14.58 & sl & 2.91 & 1.47 & 27.82 \\
\hline $0.34-0.58$ & 60.86 & 19.07 & 20.07 & scl & 3.19 & 1.50 & 28.04 \\
\hline 0.58 & Weathered gneiss mixed with soil & & & & \\
\hline
\end{tabular}


Table.4 Physico-chemical properties of groundnut-growing soils

\begin{tabular}{|c|c|c|c|c|c|c|c|c|c|c|c|c|}
\hline \multirow[t]{2}{*}{$\begin{array}{l}\text { Pedon } \\
\text { No. \& } \\
\text { Horizon }\end{array}$} & \multirow[t]{2}{*}{$\begin{array}{c}\text { Depth } \\
\text { (m) }\end{array}$} & \multicolumn{2}{|c|}{ pH $(1: 2.5)$} & \multirow[t]{2}{*}{$\underset{\left(\mathrm{dS} \mathrm{m}^{-1}\right)}{\mathrm{EC}}$} & \multirow[t]{2}{*}{$\begin{array}{c}\text { Organic } \\
\text { carbon } \\
(\%)\end{array}$} & \multirow[t]{2}{*}{$\begin{array}{c}\mathrm{CaCO}_{3} \\
(\%)\end{array}$} & \multirow[t]{2}{*}{$\begin{array}{c}\text { CEC } \\
{[\mathrm{cmol}} \\
\left.(\mathrm{p}+) \mathrm{kg}^{-1}\right]\end{array}$} & \multicolumn{4}{|c|}{$\begin{array}{c}\text { Exchangeable bases } \\
{\left[\mathrm{cmol}^{+}\left(\mathrm{p}^{+}\right) \mathrm{kg}^{-1}\right] \text { as extracted by }} \\
1 \mathrm{~N} \mathrm{NH4OAC}(\mathrm{pH} 7.0)\end{array}$} & \multirow[t]{2}{*}{$\begin{array}{c}\text { Base } \\
\text { saturat } \\
\text { ion }(\%)\end{array}$} \\
\hline & & $\begin{array}{c}\mathbf{H}_{2} \\
\mathbf{O}\end{array}$ & $\begin{array}{c}1 N \\
\mathbf{K C l}\end{array}$ & & & & & $\mathbf{C a}^{2+}$ & $\mathrm{Mg}^{+2}$ & $\mathrm{Na}^{+}$ & $\mathbf{K}^{+}$ & \\
\hline Pedon 1 & \multicolumn{12}{|c|}{ Suryanarayanapuram (Fine-loamy, smectitic, isohyperthermic, Typic Haplustept) } \\
\hline Ap & $0.00-0.20$ & 7.51 & 7.22 & 0.02 & 0.58 & 2.50 & 18.26 & 7.92 & 3.08 & 0.60 & 0.34 & 65.39 \\
\hline A1 & $0.20-0.40$ & 7.36 & 7.01 & 0.02 & 0.38 & 2.98 & 18.80 & 9.12 & 2.66 & 0.64 & 0.29 & 67.61 \\
\hline Bw1 & $0.40-0.69$ & 7.14 & 6.96 & 0.02 & 0.28 & 2.90 & 24.04 & 12.97 & 3.05 & 1.16 & 0.25 & 72.50 \\
\hline Bw2 & $0.69-0.90$ & 7.35 & 7.05 & 0.02 & 0.32 & 3.05 & 24.39 & 12.71 & & 0.92 & 0.29 & 68.72 \\
\hline Bw3 & $0.90-1.10$ & 7.66 & 7.32 & 0.01 & 0.24 & 2.90 & 24.48 & 14.14 & & 1.09 & & \\
\hline Pedon 2 & \multicolumn{12}{|c|}{ Kallivettu (Fine-loamy, smectitic, isohyperthermic, Typic Haplustept) } \\
\hline Ap & $0.00-0.18$ & 7.00 & 6.82 & 0.03 & 0.42 & 2.98 & 21.22 & 6.82 & 4.15 & 0.88 & 0.25 & 57.02 \\
\hline Bw1 & $0.18-0.55$ & 7.05 & 6.85 & 0.02 & 0.30 & 2.93 & 33.39 & 17.73 & 4.90 & 2.30 & 0.73 & 76.85 \\
\hline Bw2 & $0.55-($ & 6.91 & 6.72 & 0.02 & 0.30 & 2.90 & 24.47 & 12.18 & & 0.98 & .42 & 72.46 \\
\hline Bw3 & $0.84-1.02$ & 6.74 & 6.54 & 0.02 & 0.24 & 3.00 & 39.89 & 23.06 & 2.31 & 1.58 & 8 & 69.27 \\
\hline $\mathrm{Cr}$ & 1.02 & \multicolumn{11}{|c|}{ Weathered gneiss mixed with lime } \\
\hline Pedon 3 & \multicolumn{12}{|c|}{ Gajulapellore (Coarse-loamy, siliceous, isohyperthermic, Lithic Ustorthent) } \\
\hline Ap & $0.00-0.22$ & 6.61 & 6.51 & 0.03 & 0.42 & 2.40 & 30.31 & 12.54 & & 1.46 & .88 & 59.32 \\
\hline A1 & $.22-0.33$ & 7.02 & 6.76 & 0.02 & & 3.00 & 21.64 & 8. & & & 0 & \\
\hline $\mathbf{R}$ & 0.33 & \multicolumn{11}{|c|}{ Hard Rock } \\
\hline Pedon 4 & \multicolumn{12}{|c|}{ Kanamanambedu (Fine-loamy, kaolinitic, isohyperthermic, Typic Haplustalf) } \\
\hline Ap & $0.00-0.15$ & 7.96 & 7.45 & 0.01 & 0.42 & 2.93 & 34.58 & 18.22 & 7.84 & 1.00 & 0.44 & 79.51 \\
\hline Bt1 & $0.15-$ & 7.99 & 7.53 & 0.03 & 0.36 & 3.05 & 41.39 & 23. & & 1.62 & 8 & 88.74 \\
\hline Bt2 & $0.34-0.80$ & 8.14 & 7.55 & 0.02 & & 3.23 & 46.26 & 33.26 & & & & \\
\hline $\mathrm{Cr}$ & 0.80 & & & & & & eathered & & & & & \\
\hline Pedon 5 & \multicolumn{12}{|c|}{ Kalathuru (Fine-loamy, siliceous, isohyperthermic, Typic Ustrorthent) } \\
\hline Ap & $000-023$ & 7.14 & 6.89 & 0.04 & 0.57 & 2.90 & 12.15 & 4.9 & & & 6 & 65.02 \\
\hline A1 & & 7.05 & 6.72 & 0.02 & 0.48 & 3.05 & 18.82 & & & & & \\
\hline $\mathbf{A 2}$ & 69 & 7.22 & 6.94 & 0.02 & 0.27 & 2.93 & 33.23 & 17.30 & & 1.49 & 0.71 & 78.78 \\
\hline $\mathbf{A 3}$ & $9-0.92$ & 7.15 & 6.87 & 0.01 & 0.24 & 3.00 & 22.61 & 7.74 & 4. & 0.73 & 0.35 & 58.51 \\
\hline $\mathbf{A 4}$ & $0.92-1.30+$ & 7.44 & 7.12 & 0.01 & 0.18 & 2.93 & 36.24 & 19.48 & 7.37 & 1.43 & 0.76 & 80.13 \\
\hline Pedon 6 & \multicolumn{12}{|c|}{ Thimmasamudram (Coarse-loamy, smectitic, isohyperthermic, Typic Haplustept) } \\
\hline Ap & & 7.15 & 7.18 & 0.01 & 0.39 & 2.70 & 22.44 & 9.30 & & & & \\
\hline $\mathbf{A 1}$ & 34 & 7.31 & 7.10 & 0.02 & 0.30 & 2.65 & 23.12 & 8.91 & & & 0.37 & \\
\hline Bw1 & $4-0.84$ & 7.09 & 6.94 & 0.02 & 0.18 & 3.10 & 25.44 & 13.80 & 5. & 0.79 & 0.47 & 80.70 \\
\hline Bw2 & $0.84-1.07$ & 6.71 & 6.67 & 0.03 & 0.12 & 2.65 & 25.13 & 11.77 & 5.75 & 0.91 & 0.45 & 75.13 \\
\hline Bw3 & $1.07-1.50+$ & 7.15 & 6.94 & 0.01 & 0.09 & 2.60 & 30.67 & 14.24 & 10.0 & 1.22 & 0.69 & 85.39 \\
\hline Pedon 7 & \multicolumn{12}{|c|}{ Chittathur (Coarse-loamy, siliceous, isohyperthermic, Typic Ustrorthent) } \\
\hline Ap & $0.00-0.22$ & 7.71 & 7.43 & 0.01 & 0.48 & 2.78 & 18.13 & 7.1 & 4. & 2.22 & 0.72 & 78.65 \\
\hline $\mathbf{A 1}$ & $0.22-0.46$ & 7.04 & 6.89 & 0.02 & 0.31 & 2.90 & 18.82 & 7.8 & & 1.31 & 0.48 & 80.71 \\
\hline $\mathbf{A 2}$ & $0.46-0.81$ & 6.97 & 6.82 & 0.02 & 0.20 & 2.73 & 13.07 & 3.90 & 2.50 & 0.60 & 0.31 & 55.93 \\
\hline $\mathrm{Cr}$ & 0.81 & \multicolumn{11}{|c|}{ Weathered gneiss mixed with soil } \\
\hline Pedon 8 & \multicolumn{12}{|c|}{ Brahmanapalle (Coarse-loamy, siliceous, isohyperthermic, Typic Ustrorthent) } \\
\hline Ap & $0.00-0.16$ & 6.70 & 6.54 & 0.01 & 0.46 & 3.00 & 19.33 & 8.19 & 2.3 & 1.75 & 0.69 & 67.20 \\
\hline A1 & $5-0.34$ & 7.24 & 7.12 & 0.01 & 0.32 & 2.65 & & & & 1.26 & 0.49 & 64.31 \\
\hline $\mathbf{A 2}$ & $0.34-0.58$ & 7.73 & 7.34 & 0.02 & 0.18 & 2.75 & 18.92 & 7.09 & 3.9 & 1.46 & 0.64 & 69.56 \\
\hline $\mathrm{Cr}$ & 0.58 & & & & & eatherec & gneiss $n$ & ed with & & & & \\
\hline
\end{tabular}


Table.5 Available nutrient status of the groundnut-growing soils ( $\mathrm{mg} \mathrm{kg}^{-1}$ )

\begin{tabular}{|c|c|c|c|c|c|c|c|c|c|}
\hline \multirow{2}{*}{$\begin{array}{c}\text { Pedon } \\
\text { No. \& } \\
\text { Horizon } \\
\end{array}$} & \multirow{2}{*}{$\begin{array}{c}\text { Depth } \\
\text { (m) }\end{array}$} & \multicolumn{4}{|c|}{ Available macronutrients } & \multicolumn{4}{|c|}{ Available micronutrients } \\
\hline & & $\mathbf{N}$ & $\mathbf{P}$ & $\mathbf{K}$ & $\mathbf{S}$ & $\mathbf{Z n}$ & $\mathbf{C u}$ & Fe & Mn \\
\hline Pedon 1 & \multicolumn{9}{|c|}{ Suryanarayanapuram (Fine-loamy, smectitic, isohyperthermic, Typic Haplustept) } \\
\hline Ap & $0.00-0.20$ & 106.40 & 11.55 & 84.71 & 26.35 & 0.69 & 1.53 & 27.33 & 3.84 \\
\hline $\mathbf{A 1}$ & $0.20-0.40$ & 89.60 & 10.70 & 77.23 & 18.76 & 0.33 & 1.21 & 7.98 & 5.80 \\
\hline Bw1 & $0.40-0.69$ & 84.00 & 9.20 & 94.54 & 16.27 & 0.70 & 1.17 & 5.90 & 2.06 \\
\hline Bw2 & $0.69-0.90$ & 77.20 & 7.45 & 83.54 & 11.00 & 0.75 & 0.75 & 5.14 & 5.80 \\
\hline Bw3 & $0.90-1.10$ & 72.80 & 6.35 & 45.46 & 7.31 & 0.54 & 0.62 & 5.87 & 3.20 \\
\hline Pedon 2 & \multicolumn{9}{|c|}{ Kallivettu (Fine-loamy, smectitic, isohyperthermic, Typic Haplustept) } \\
\hline$\overline{A p}$ & $0.00-0.18$ & 185.10 & 14.50 & 144.07 & 29.80 & 0.80 & 1.36 & 24.30 & 13.74 \\
\hline Bw1 & $0.18-0.55$ & 127.20 & 14.00 & 112.00 & 16.82 & 0.83 & 1.48 & 12.87 & 24.78 \\
\hline Bw2 & $0.55-0.84$ & 120.40 & 15.23 & 70.46 & 14.82 & 0.44 & 2.01 & 6.82 & 16.80 \\
\hline Bw3 & $0.84-1.02$ & 112.40 & 15.80 & 57.94 & 9.20 & 0.91 & 1.95 & 8.25 & 24.54 \\
\hline $\mathrm{Cr}$ & 1.02 & \multicolumn{8}{|c|}{ Weathered gneiss mixed with lime } \\
\hline Pedon 3 & \multicolumn{9}{|c|}{ Gajulapellore (Coarse-loamy, siliceous, isohyperthermic, Lithic Ustorthent) } \\
\hline $\mathbf{A p}$ & $0.00-0.22$ & 129.60 & 14.27 & 116.53 & 17.15 & 2.07 & 3.52 & 96.54 & 67.46 \\
\hline$\overline{\mathrm{A1}}$ & $0.22-0.33$ & 105.20 & 12.43 & 98.15 & 11.50 & 0.53 & 2.93 & 21.12 & 14.96 \\
\hline $\mathbf{R}$ & 0.33 & \multicolumn{8}{|c|}{ Hard Rock } \\
\hline Pedon 4 & \multicolumn{9}{|c|}{ Kanamanambedu (Fine-loamy, kaolinitic, isohyperthermic, Typic Haplustalf) } \\
\hline Ap & $0.00-0.15$ & 122.00 & 9.67 & 113.17 & 39.80 & 0.64 & 1.42 & 3.63 & 16.20 \\
\hline Bt1 & $0.15-0.34$ & 114.40 & 12.50 & 100.29 & 32.50 & 0.42 & 1.29 & 4.99 & 18.60 \\
\hline Bt2 & $0.34-0.80$ & 95.20 & 10.70 & 54.57 & 19.50 & 0.87 & 1.01 & 5.45 & 33.66 \\
\hline $\mathrm{Cr}$ & \multicolumn{9}{|c|}{ Weathered gneiss } \\
\hline Pedon 5 & \multicolumn{9}{|c|}{ Kalathuru (Fine-loamy, siliceous, isohyperthermic, Typic Ustrorthent) } \\
\hline Ap & $0.00-0.23$ & 178.40 & 12.70 & 138.73 & 33.30 & 1.30 & 3.61 & 28.72 & 76.28 \\
\hline$\overline{\mathbf{A 1}}$ & $0.23-0.44$ & 167.00 & 11.41 & 127.83 & 24.10 & 0.98 & 3.57 & 7.64 & 23.38 \\
\hline $\mathbf{A 2}$ & $0.44-0.69$ & 116.40 & 10.13 & 123.55 & 20.76 & 0.91 & 1.68 & 45.55 & 55.90 \\
\hline $\mathbf{A 3}$ & $0.69-0.92$ & 112.00 & 8.33 & 76.72 & 18.65 & 0.91 & 1.70 & 15.42 & 27.20 \\
\hline $\mathbf{A 4}$ & $0.92-1.30+$ & 98.80 & 8.67 & 57.02 & 11.20 & 0.82 & 1.31 & 12.69 & 17.74 \\
\hline Pedon 6 & \multicolumn{9}{|c|}{ Thimmasamudram (Coarse-loamy, smectitic, isohyperthermic, Typic Haplustept) } \\
\hline $\mathbf{A p}$ & $0.00-0.20$ & 107.60 & 10.33 & 111.95 & 20.41 & 1.08 & 2.58 & 36.52 & 35.76 \\
\hline$\overline{\mathrm{A1}}$ & $0.20-0.54$ & 96.40 & 9.33 & 98.20 & 15.26 & 0.86 & 1.37 & 52.60 & 78.85 \\
\hline Bw1 & $0.54-0.84$ & 90.40 & 9.00 & 155.53 & 18.50 & 1.02 & 1.45 & 95.75 & 48.80 \\
\hline Bw2 & $0.84-1.07$ & 74.80 & 7.67 & 95.45 & 10.50 & 1.22 & 1.89 & 12.97 & 12.75 \\
\hline Bw3 & $1.07-1.50+$ & 58.80 & 7.33 & 283.36 & 11.00 & 1.50 & 1.76 & 23.10 & 23.47 \\
\hline Pedon 7 & \multicolumn{9}{|c|}{ Chittathur (Coarse-loamy, siliceous, isohyperthermic, Typic Ustrorthent) } \\
\hline$\overline{A p}$ & $0.00-0.22$ & 95.20 & 9.33 & 112.46 & 17.95 & 0.76 & 2.05 & 7.00 & 20.34 \\
\hline$\overline{\mathbf{A 1}}$ & $0.22-0.46$ & 84.00 & 8.00 & 146.35 & 11.73 & 0.65 & 1.81 & 5.32 & 13.31 \\
\hline $\mathbf{A 2}$ & $0.46-0.81$ & 78.40 & 7.50 & 178.95 & 18.71 & 1.07 & 1.95 & 5.79 & 11.32 \\
\hline $\mathrm{Cr}$ & 0.81 & \multicolumn{8}{|c|}{ Weathered gneiss mixed with soil } \\
\hline Pedon 8 & \multicolumn{9}{|c|}{ Brahmanapalle (Coarse-loamy, siliceous, isohyperthermic, Typic Ustrorthent) } \\
\hline$\overline{A p}$ & $0.00-0.16$ & 121.20 & 7.67 & 81.90 & 19.76 & 0.53 & 1.48 & 18.59 & 6.91 \\
\hline$\overline{\mathbf{A 1}}$ & $0.16-0.34$ & 105.20 & 5.00 & 73.56 & 5.58 & 0.34 & 0.97 & 15.03 & 5.11 \\
\hline$\overline{\mathbf{A 2}}$ & $0.34-0.58$ & 88.40 & 4.33 & 59.36 & 11.30 & 0.32 & 0.56 & 19.61 & 7.19 \\
\hline $\mathrm{Cr}$ & 0.58 & \multicolumn{8}{|c|}{ Weathered gneiss mixed with soil } \\
\hline
\end{tabular}


The lower phosphorus content in sub-surface horizons could be attributed to the fixation of released phosphorus by clay minerals and oxides of iron and aluminium. The available $\mathrm{K}$ in soils of different sites was low to high and ranged from 54.57 to $283.36 \mathrm{mg} \mathrm{kg}^{-1}$. The available potassium in all the horizons of the pedons was found to be low to high. Slow weathering of mica and fixation of released potassium might have resulted in low exchangeable potassium status (Prakash and Rao, 2002). The higher potassium could be attributed to more intense weathering, release of liable $\mathrm{K}$ from organic residues, application of $\mathrm{K}$ fertilizers and upward translocation of potassium from lower depths along with capillary raise of ground water. Similar results were reported by Reddy and Naidu (2016) in soils of Chennur mandal of Kadapa district in Andhra Pradesh. The available sulphur in soils was high and varied from 5.58 to 39.80 $\mathrm{mg} \mathrm{kg}^{-1}$. Due to higher organic matter in surface layers than in deeper layers, the available sulphur is more in surface horizons than in the sub-surface horizons.

\section{Micronutrients}

These soils had relatively low zinc as compared to copper, iron and manganese. The DTPA extractable $\mathrm{Zn}$ ranged from 0.32 to $1.49 \mathrm{mg} \mathrm{kg}{ }^{-1}$ soil. The groundnut-growing soils were sufficient in surface horizons and sub-surface horizons in all pedons except in pedons 1, 2, 3 and 4 where in, it was found to deficient in sub-surface horizons. The relatively high content of zinc in surface horizons may be attributed to variable intensity of the pedogenetic processes and more complexing with organic matter, which resulted in chelating of $\mathrm{Zn}$ (Verma et al., 2005). The DTPA extractable Fe content varied from 3.63 to $96.54 \mathrm{mg} \mathrm{kg}^{-1}$. According to critical limit of $4.5 \mathrm{mg} \mathrm{kg}^{-1}$ of Lindsay and Norvell (1978), the soils were sufficient in available iron (expect P4 of Ap Horizon). The higher concentration of DTPA-Fe in Ap horizons of the pedons may be due to accumulation of organic carbon in the surface horizons. The organic carbon due to its affinity to influence the availability of iron by chelating action might have protected the iron from oxidation and precipitation, which consequently increased the availability of iron in the surface horizons (Prasad and Sakal, 1991). All the pedons were found to be sufficient in available copper and manganese as all the values were well above the critical limit of $0.2 \mathrm{mg} \mathrm{kg}^{-1}$ and $1.0 \mathrm{mg} \mathrm{kg}^{-1}$, respectively (Lindsay and Norvell (1978).

\section{Soil classification}

Pedons 1, 2, and 6 had cambic epipedon in sub-surface horizon and hence these are grouped in order Inceptisol. Owing to ustic moisture regime in the area, these pedons have been classified as Ustepts at sub-order level. The soils do not have duripan, calcic / petrocalcic horizon within $100 \mathrm{~cm}$ and less than 60 per cent BSP with in $25 \mathrm{~cm}$ and 75 $\mathrm{cm}$ from the mineral soil surface and hence grouped under great group Haplustepts. The pedons 1,2 and 6 did not exhibit intergradation with other taxa or an extragradation from the central concept. Hence, these pedons were logically classified as Typic Haplustepts at sub-group level with coarse loamy (P6) and fine-loamy ( $\mathrm{P}_{1}$ and $\mathrm{P} 2$ ) textural family classes as these pedons had less than 18 per cent clay ( silt $+1 \frac{1}{2} 2$ times clay $\leq 15$ ) and high sand content (weighted average $>85 \%$ ) and more than 18 per cent and less than 35 per cent clay (weighted average) in the control section, respectively. These pedons have isohyperthermic temperature regime and smectitic mineralogy. The presence of more smectite content by weight than any other single kind of charge mineral qualifies smectitic mineralogy. The 
pedons 3, 5, 7 and 8 does not meet the requirement of orders, further it does not meet the requirement of sub-order Arents, Psamments, Fluvents and hence keyed out as Orthents. Because of presence of ustic moisture regime, these pedons have been classified under great group Ustorthents.

As these pedons do not have lithic contact (expect Pedon 3) within $50 \mathrm{~cm}$ of mineral layer and no cracks within $125 \mathrm{~cm}$ of the mineral surface that are $5 \mathrm{~mm}$ or more wide to a thickness of $30 \mathrm{~cm}$ or more and linear extensibility of $6 \mathrm{~cm}$ or more, hence placed under Typic Ustorthent at sub-group level. Pedon 3 having lithic contact within $50 \mathrm{~cm}$ of the mineral soil surface, due to this classified as Lithic Ustorthent.

Pedon 4 showed the presence of diagnostic argillic (Bt) sub-surface horizon as evidenced by the fact that illuvial horizon contained 1.2 times more clay than eluvial horizon and also had base saturation more than $35 \%$ throughout the profile. However, this pedon was classified as Ustalf at sub-order level due to presence of ustic moisture regime. Pedon 4 did not have duripan, plinthite, kandic, natric or petrocalcic horizons and the argillic horizon did not exhibit a hue of 2.5 YR; hence pedon 4 was logically classified as Haplustalf at great group level.

Finally the pedon 4 was classified under Typic Haplustalfs at sub-group level due to absence of lithic or paralithic contact, cracks, pumice like fragments, volcanic ash, lamelle and calcic horizon and also an argillic horizon with a base saturation (by sum of cations) of less than 75 per cent. This pedon has more than 18 per cent and less than 35 per cent clay (weighted average) in the control section and hence classified under fine-loamy class with kaolinitic mineralogy.

\section{References}

AIS and LUS 1970. Soil Survey Manual. All India Soil and Land Use Survey Organisation. IARI, New Delhi. pp. 163.

Finck, A and Venkateswarulu, J. 1982. Chemical properties and fertility management of Vertisols. In vertisols and rice soils in the tropics. Symposia of $12^{\text {th }}$ International Congress of Soil Science, New Delhi held on 8-16. Feb.1982.

Lindsay, W.L and Norvell, W.A. 1978. Development of DTPA soil test for zinc, iron, manganese and copper. Soil Science Society of American Journal. 43: 421-428.

Muhr, G.R., Datta, N.P., Sankarasubramoney, H., Laley, V.K and Donahue, R.L. 1965. Critical soil test values for available $\mathrm{N}, \mathrm{P}$ and $\mathrm{K}$ in different soils. In soil testing in India. 2nd edition, USAID mission to India, New Delhi. 52-56.

Prakash, T.R and Rao, M.S. 2002. Characterization and classification of some soils in a part of Krishna district, Andhra Pradesh. The Andhra Agricultural Journal. 49: 228-236.

Prasad, S.N and Sakal, R. 1991. Availability of iron in calcareous soils in relation to soil properties. Journal of the Indian Society of Soil Science. 39: 658-661.

Rao, I.V.Y.R and Sunil, K.B.G. 2010. Sugarcane production and productivity trends in Andhra Pradesh during last three decades with special reference to north coastal districts of Andhra Pradesh. $24^{\text {th }}$ meeting of Sugarcane research and development workers of Andhra Pradesh. Souvenir.

Ray, S.K and Reddy R.S. 1997. Quarternary soil formations in some Godavari delta region. Journal of the Indian Society of Soil Science 45, 208-211. 
Reddy, K.S and Naidu, M.V.S. 2016. Characterization and classification of soils in semi-arid region of Chennur mandal in Kadapa district, Andhra Pradesh. Journal of the Indian Society of Soil Science. 64(3): 207-217.

Six, J., Elliott, E.T, Paustian, K and Doran, J.W. 1998. Aggregation and soil organic matter accumulation in cultivated and native grass land soils. Soil Science of America Journal. 62: 1367-1377.

Soil Survey Staff 1951. Soil Survey Manual US Department of Agricultural Hand book No. 18.

Soil Survey Staff. 2014. Keys to soil taxonomy (Twelveth edition), USDA,
Natural Resource Conservation Service, Washington, DC.

Tandon, H.L.S. 1991. Sulphur research and agricultural production in India. $3^{\text {rd }}$ edition, The Sulphur Institute, Washington, D C. 140-148.

Verma, V.K., Setia, R.K., Sharma, P.K, Charanjit Singh and Ashok Kumar 2005. Micronutrient distribution in soils developed on different physiographic units of Fatehgrah Sahib district of Punjab. Agropedology. 15(1): 70-75.

Zonn, S.V. 1986. Tropical and Sub-tropical Soil Science (translated from the Russian), Mir Publishers, Moscow, p. 260.

\section{How to cite this article:}

Nagarjuna, V. and Naidu, M.V.S. 2018. Characterization, Classification and Nutritional Status of Groundnut Growing Soils in Southern Agro-Climatic Zone: A Case Study on Western Mandals of Srikalahasti Division in Chittoor District, Andhra Pradesh, India. Int.J.Curr.Microbiol.App.Sci. 7(05): 751-762. doi: https://doi.org/10.20546/ijcmas.2018.705.092 